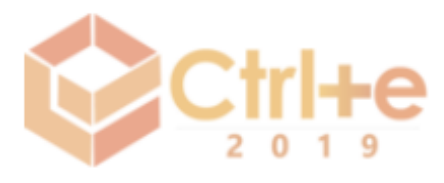

IV Congresso sobre Tecnologias na Educação (Ctrl+E 2019)

Recife, Pernambuco - Brasil

28 a 30 de agosto de 2019

\title{
Desenvolvimento de um Protótipo de Identificação do Nível de Escrita para Dispositivos Móveis: uma Ferramenta de Sondagem no Ensino
}

\author{
Claudemir J Lima1, Elidiene G. Oliveira, Gustavo Callou, Gilberto A. de A. \\ Cysneiros F.1 \\ 1Departamento de Informática - Universidade Federal Rural de Pernambuco (UFRPE) - \\ Recife - PE - Brasil \\ \{claudemir.jlima, gustavo.callou, gilberto.cysneiros\}@ufrpe.br, \\ elidienegomes@gmail.com
}

\begin{abstract}
The poll of the student's writing level is very important for the teacher to direct his practices with activities that promote the advancement of writing. There are few applications that perform poll of the writing level, so this article describes the creation of an NEA (Alphabetic Writing Level) prototype to perform a poll of the writing level in the literacy process that is based on the Psychogenesis of written language. The partial results of the prototype, according to the teacher member of a municipal school of the public network of Recife, contributed to the construction of an instrument for the diagnostic evaluation of the students' writing level. The partnership between the professional in the pedagogical and technological area for creating the educational application is essential.
\end{abstract}

\begin{abstract}
Resumo. A sondagem do nível de escrita do estudante é muito importante para o professor direcionar suas práticas com atividades que promovam o avanço da escrita. Há poucos aplicativos que realizam a sondagem do nível de escrita, dessa forma, esse artigo descreve a criação de um protótipo NEA (Nivel de Escrita Alfabética) para realização de sondagem do nível de escrita no processo de alfabetização que é embasado na Psicogênese da língua escrita. Os resultados parciais do protótipo, segundo o professor participante de uma escola municipal da rede pública do Recife, contribuíram para a construção de um instrumento de avaliação diagnóstica do nível de escrita dos estudantes. A parceria do profissional na área pedagógica e tecnológica para criação do aplicativo educacional está sendo essencial.
\end{abstract}

\section{Introdução}

É notória a necessidade da inserção tecnológica na escola como meio que contribui para ação pedagógica no processo de ensino e aprendizagem. A importância da intervenção dessas ações é fundamental para realização de um trabalho mais estratégico e eficaz na instituição de ensino. São diversos recursos tecnológicos que facilitam o acesso ao conhecimento e cada vez mais a sociedade está imersa nesses meios (DEMO 2010b, 
SILVA, 2017). Assim sendo, com o aumento da convergência multididática em tecnologias móveis, juntamente com a parceira multidisciplinar de profissionais na área da educação e tecnologia, ampliam-se as possibilidades de construção de aplicativos que auxiliem as diversas necessidades educacionais.

O conhecimento tecnológico para desenvolvimento de aplicativos para Android vem crescendo para serem utilizados nos dispositivos móveis como tablets e celulares. Muitas escolas utilizam o tablet em suas atividades pedagógicas em sala de aula, além disso os estudantes vêm apresentando cada vez mais habilidades no uso de aplicativos Android.

Dentro desse objetivo essa pesquisa visa conhecer a linguagem de programação Kotlin, analisar a funcionalidade do aplicativo com parceria do conhecimento de um professor alfabetizador, validar a aplicabilidade e possíveis melhorias do aplicativo. A metodologia da pesquisa incidirá a partir de um Estudo de Caso de caráter descritivo numa abordagem qualitativa a partir de um estudo teórico, do aplicativo em desenvolvimento, da observação estruturada de participantes e através de entrevistas semi-estruturadas. Os participantes serão o professor alfabetizador e estudantes em processo de alfabetização dos anos iniciais do ensino fundamental.

\section{Tecnologia na Educação}

As Tecnologias de Informação e Comunicação (TICs), contribuem na educação, ampliando as possibilidades no processo de ensino e aprendizagem. Para Kenski (2008) e Moran (2007) os recursos tecnológicos são significativos para o processo de ensino e aprendizagem se forem utilizados de forma planejada, lógica e organizada.

Segundo Moran (2004) "as tecnologias sozinhas não mudam a escola, mas trazem mil possibilidades de apoio ao professor e de interação com e entre os alunos". Dessa forma, é preciso do apoio do professor juntamente com o estudante para conhecer as necessidades educacionais da escola, utilizando os recursos de forma mais eficaz e significativa. Assim sendo, surgem crescentemente recursos digitais como aplicativos educacionais com diferentes visões metodológicas para atender diversas necessidades educacionais.

É necessário dessa maneira, conhecer os aplicativos e recursos disponíveis para que se possam usá-los com a estratégia mais adequada de interação entre estudante, professor e as (TICs). Nesse contexto, o desenvolvedor de aplicativos necessita se articular com a equipe pedagógica para que de forma multidisciplinar possa desenvolver pesquisas com o uso das tecnologias da informação, de forma que atenda às necessidades educacionais contribuindo na construção do conhecimento.

\section{Sondagem do Sistema de Escrita Alfabética}

Há uma necessidade para que o professor possa avaliar o nível de escrita alfabética da criança, jovens ou adultos em processo de alfabetização. Esse sistema de escrita alfabética é baseado em teorias que classificam o nível de escrita. Essas teorias contribuem para a prática dos professores alfabetizadores adequando suas metodologias e estratégias baseada na sondagem de escrita do estudante e analisando o raciocínio que a criança faz para escrever e compreender o processo de construção do sistema de escrita alfabética (SEA).

Uma das teorias da escrita é embasada segundo a teoria da Psicogênese da língua 
escrita desenvolvida por Ferreiro e Teberosky (1999). A autora Emília Ferreiro classifica os níveis de escrita em: pré-silábico, silábico, silábico- alfabético e alfabético. Artur Moraes em concordância com Magda Soares também classifica a fases de escrita alfabética. Morais et al. (2012) afirmam que para analisar o nível de escrita das crianças é preciso:

Um dos programas desenvolvido pelo MEC que é o Pacto Nacional da Educação na Idade Certa com Artur Morais, Telma Ferraz. Outros municípios também trabalham com o projeto do Alfaletrar com Magda Soares todos voltados para o avanço na educação se baseando na teoria da Psicogênese da língua escrita desenvolvida por Ferreiro e Teberosky (1999).

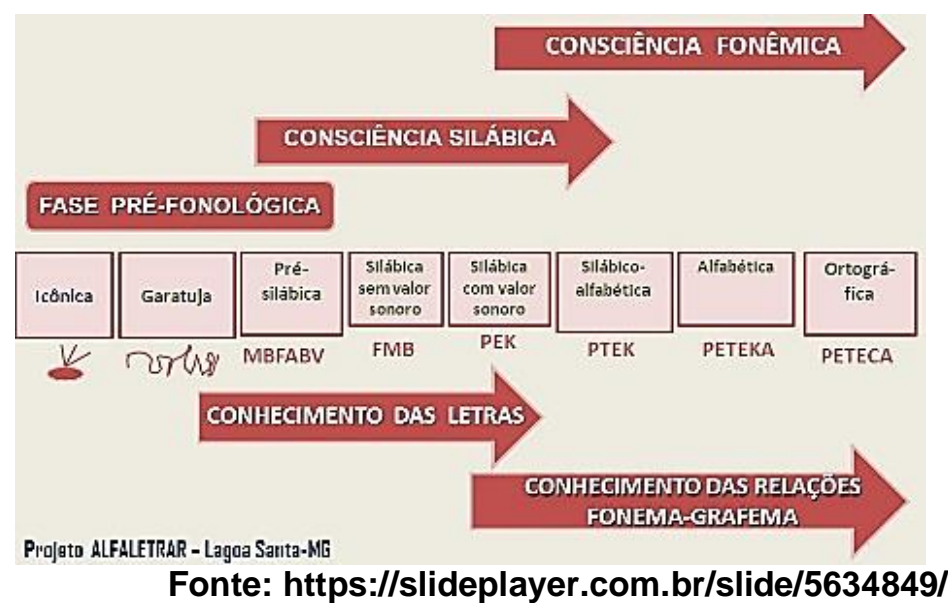

Como se observa na imagem do projeto ALFALETRAR, desenvolvido por Magda Soares as crianças passam por algumas fases de escrita na qual o professor precisa realizar avaliações diagnósticas, para acompanhar a evolução do estudante e organizar atividades para o avanço da fase de escrita.

Dessa forma essa pesquisa propõe o desenvolvimento de tecnologia aplicada para colaborar com a prática pedagógica do professor na realização dessa sondagem do nível do sistema de escrita alfabética (SEA). Baseado nessa necessidade foi realizado um protótipo de um aplicativo para Android com a linguagem de programação Kotlin para sondagem do nível de escrita do estudante chamado de NEA- Nível de Escrita Alfabética. Entretanto, foi pesquisado alguns artigos, aplicativos para dispositivo móveis e aplicativos para web. Tais pesquisas foram abordadas no seguinte tópico.

\section{Trabalhos relacionados ao protótipo do aplicativo NEA}

São poucos os trabalhos encontrados sobre aplicativos para sondagem do nível de escrita até o momento dessa pesquisa, no entanto, foi possível ler os artigos publicados e entender a aplicabilidade e funcionalidades dos aplicativos. Dentre eles, esse artigo se baseou nos seguintes trabalhos: Psicogênese da escrita infantil - PEI, ROSSETTO (2017), Aplicativo para Auxiliar Professores nas Avaliações de Psicogênese da Escrita, MOMBACH et al. (2017) e o Aplicativo Sondagem Descomplicada: sistema de informação aplicado à alfabetização, BORGES et al. (2016). A busca por trabalhos baseados nessa tese no Portal de Publicações da CEIE mostra que o tema ainda precisa de maior relevância na área de Informática na Educação no Brasil, visto que a quantidade de pesquisas é limitada (MOMBACH et al. 2017). Dentre eles foram citados os seguintes artigos: 


\subsection{Psicogênese da escrita infantil - PEI, ROSSETTO (2017), Alessandra.}

A pesquisadora desse primeiro protótipo do aplicativo PEI contou com o apoio de uma plataforma gratuita Fábrica de Aplicativos (2017). No entanto, a mesma necessitou do auxílio de um programador para dar continuidade ao protótipo pois a plataforma Fábrica de Aplicativos não oferecia condições de avançar para evolução do aplicativo. Dessa forma a ferramenta online Marvell App e Google Firebase, Software Development Kit (SDK), ou seja, um conjunto de ferramentas foram utilizadas. As seguintes atividades foram realizadas:

- Projetar as telas, definir cores;

- Criar os módulos que fariam parte de cada aba do aplicativo;

- Criar a logomarca;

- Desenvolver os algoritmos que se aproximariam ao máximo da leitura real da escrita das crianças;

- Inserir a parte auditiva;

- Buscar por imagens e áudios compatíveis com o campo semântico escolhido.

\subsection{Aplicativo para Auxiliar Professores nas Avaliações de Psicogênese da Escrita,} MOMBACH et al. (2017)

O presente aplicativo foi desenvolvido com apoio dos recursos da Unified Modeling Language (UML) e do Massachusetts Institute of Technology (MIT) App Inventor 3 para o sistema Android 4. Tal aplicativo permite:

- Que os professores avaliem a habilidade de escrita dos alunos;

- Que os professores adicionem novas atividades de acordo com o nível de escrita do estudante;

- Analisar o progresso dos alunos com base em testes previamente aplicados.

\subsection{Sondagem Descomplicada: sistema de informação aplicado à alfabetização, BORGES et al. (2016)}

Esse aplicativo utilizou como armazenamento o banco de dados do Android (SQLite) e também o auxílio do Google Vision para pode detectar conjuntos de objetos em suas imagens chamada tecnologia Optical Character Recognition (OCR), a partir de flores, animais ou transporte pois o Google Vision detecta e extrai o texto dentro da imagem, para uma grande variedade de línguas, juntamente com suporte para a identificação automática de idiomas (GOOGLE, 2016, online apud BORGES et al., 2016). Nele houve a inserção dos seguintes dados:

- Palavras escritas do estudante (monossílaba, dissílaba, trissílaba e polissílaba) e uma frase com uma das palavras escritas para cadastro de sondagem e sugestão da fase de escrita do estudante;

- Automatização na separação de sílabas;

- Captura e armazenamento de imagens das escritas;

- Gráficos de evolução de cada aluno no processo de sondagem, consulta, alteração e remoção de registros;

- Consultas de sondagens;

- Modelos de sondagens de acordo com o campo semântico de imagens que faz parte da mesma categoria, exemplo: lista de animais, lista de doces.

\subsection{Protótipo NEA}


Para o desenvolvimento do protótipo utilizando a ferramenta Android Studio, com as linguagens de programação Java e Kotlin. Para o armazenamento dos dados foi utilizado o Firebase da Google. O protótipo permite:

- Inserir a logomarca;

- Desenvolver uma função que se aproxima ao máximo da hipótese de escrita da escrita das crianças;

- Inserir imagens vinculadas com lista de palavras do mesmo campo semântico

- Exibir feedback para cada palavra respondida pelo estudante

- Exibir sugestão da hipótese do nível de escrita do estudante

\section{Metodologia}

Sendo de caráter descritivo, essa pesquisa dispõe-se a interagir com o professor participante para uma melhor compreensão da sondagem do nível de escrita do estudante em processo de alfabetização, independente da faixa etária. O professor nessa versão, faz a simulação de algumas possibilidades de hipótese de escrita do estudante para o aplicativo sugerir o nível de escrita. Após a simulação e análise do professor, organizouse uma lógica de programação para o desenvolvimento do aplicativo NEA. Portanto, é essencial compreender a realidade com suas especificidades e influências em diferentes fenômenos que não podem ser calculados e sim compreendidos aproximando mais a realidade com o objeto de investigação. (ZANETTE, 2017, p. 157).

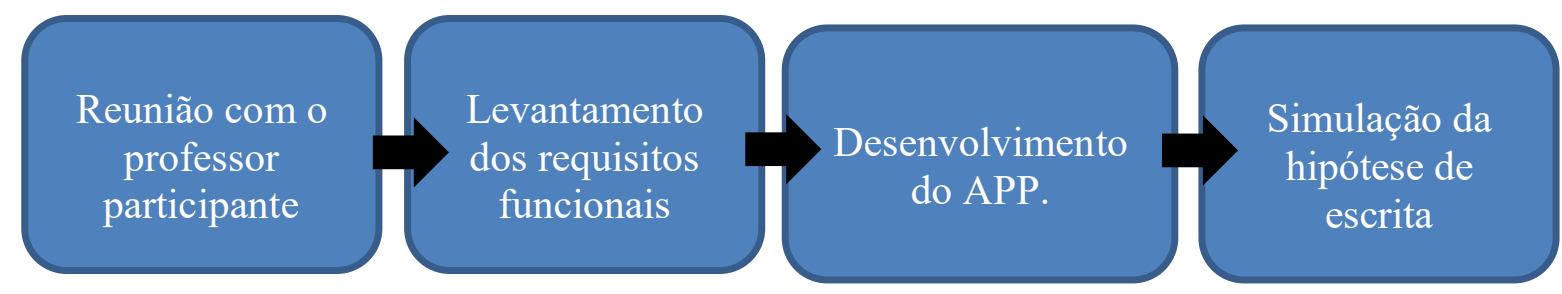

Figura 1. Etapas da metodologia

5.1- Reunião com o professor formador: Foi realizado reunião para entendimento da dinâmica no tocante ao método utilizado para avaliar o nível da escrita.

5.2- Levantamento dos requisitos funcionais: Nesta etapa foi identificado os requisitos funcionais do aplicativo e formatado a ordem de desenvolvimento das telas do aplicativo.

5.3- Desenvolvimento do app (protótipo): Para o desenvolvimento foi utilizado o Android Studio, com as linguagens de programação Java e Kotlin. Para o armazenamento dos dados utilizamos o Firebase da Google.

5.4- Simulação da hipótese de escrita: Após o protótipo desenvolvido o professor alfabetizador realizou a instalação do app no dispositivo móvel. Em seguida, simulou a escrita dos estudantes nas fases de hipótese disponíveis no aplicativo NEA tais como: présilábico, silábico sem valor sonoro, silábico com valor sonoro, silábico-alfabético e alfabético. 


\section{Resultados Preliminares}

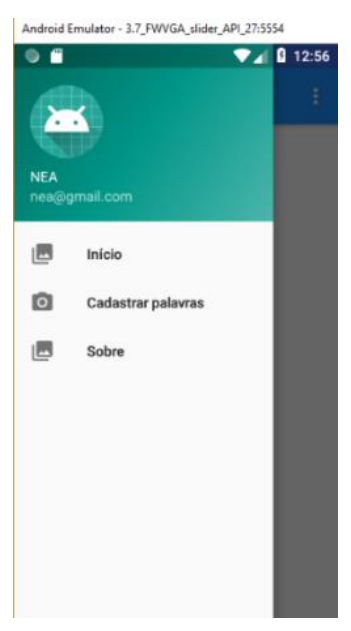

Tela 01

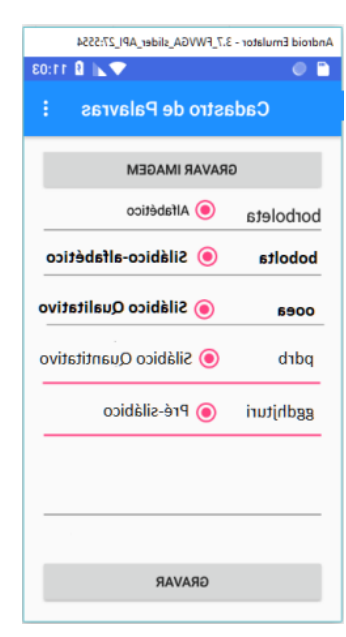

Tela 02

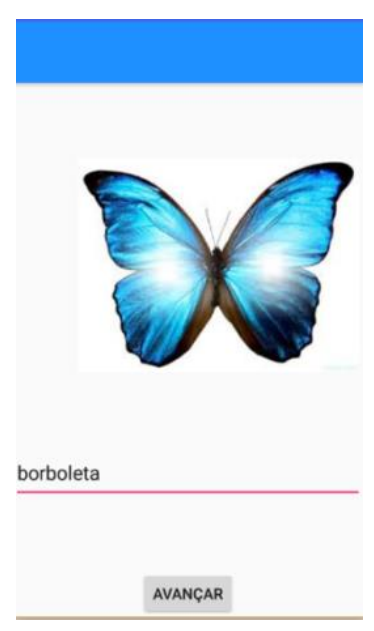

Tela 03

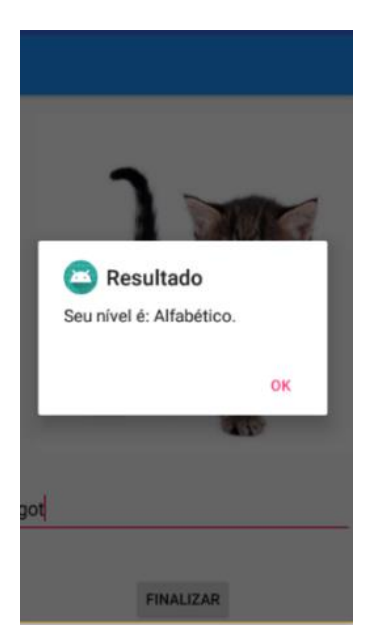

Tela 04

Figura 2. Telas do aplicativo

Após o modelo criado pelo professor simulando as possibilidades de hipótese de escrita do estudante para cada palavra inserida, percebeu-se qual seriam os requisitos funcionais para o desenvolvimento do protótipo. O professor solicitou que fossem listadas palavras do mesmo campo semântico sendo exibida uma imagem em cada formulário, representando a palavra analisada pelo app, como mostrado na terceira e quarta tela da Figura 2. Para facilitar a visualização foi solicitado o uso de imagens reais para cada palavra. A primeira tela da Figura 2 exibe um menu contendo os itens: "Início", "Cadastrar palavras" e "Sobre". A segunda tela da Figura 2 permite que o usuário professor, cadastre imagens e palavras que serão utilizadas para a avaliação do nível de escrita. Após a inserção da palavra o professor digitará logo abaixo as possíveis respostas e classificará o seu nível da escrita, assim o aplicativo irá comparar com o digitado pelo aluno como mostra na terceira tela da Figura 2.

Após concluir o cadastro das palavras o professor clicará no menu iniciar e aparecerá a terceira tela mostrada na Figura 2, quando o aluno digitar a palavra e clicar no botão Avançar o app irá para a próxima tela com uma nova imagem e campo para inserção da digitação pelo aluno. Após a digitação e validação de todas as palavras, o aplicativo irá sugerir o resultado da sondagem conforme mostrado na quarta tela da Figura 2. Após a análise do funcionamento do aplicativo quando o professor fez a simulação da hipótese de escrita do estudante, o professor percebeu que o aplicativo necessitava de ajuste para classificar alguns níveis corretamente.

\section{Considerações Finais}

O diálogo com o professor alfabetizador sobre a necessidade de criação de um aplicativo para apoiá-lo na sondagem de escrita alfabética foi essencial. Os testes realizados por ele no app foram significativos para analisar se a sugestão dos mesmos classificava corretamente o nível de escrita do estudante para buscar futuros ajustes. Desta forma, foi possível fazer uma parceira multidisciplinar com profissional da educação e da tecnologia no desenvolvimento do aplicativo e validar a aplicabilidade com possíveis melhorias e 
posteriormente serem testadas com os estudantes. Para continuidade do desenvolvimento do protótipo sugere-se que o resultado também armazene em banco de dados Firebase um histórico (portfólio) de respostas de cada estudante. Assim sendo, contribuindo para auxiliar o professor na hipótese de escrita de cada estudante. Com isso essa pesquisa busca contribuir para futuros estudos envolvendo o desenvolvimento de aplicativos para dispositivos móveis com a finalidade de fazer sondagem da hipótese de escrita alfabética, ampliando as possibilidades de recursos pedagógicos com o uso das tecnologias na educação.

\section{Referências}

BORGES, Fábio Ferreira; Oliveira, PATRICK Mendonça; PIRES, Daniel Facciolo. Sondagem Descomplicada: sistema de informação aplicado à alfabetização. Revista Eletrônica de Sistema de Informação e Gestão Tecnológica. Vol.07,n.01-2016. Disponível em: http://periodicos.unifacef.com.br/index. php/resiget/issue/view/130 Acesso em: 16 de junho de 2018

KENSKI, Vani Moreira. A urgência de propostas inovadoras para a formação de professores para todos os níveis de ensino. Rev. Diálogo Educ., Curitiba, v. 15, n. 45, p. 423-441, maio/ago. 2015.

MORAN, José Manuel. A educação que desejamos: novos desafios e como chegar lá. 2. ed.Campinas, SP: Papirus, 2007. 174p.

MORAN, José Manuel. OS NOVOS ESPAÇOS DE ATUAÇÃO DO PROFESSOR COM AS TECNOLOGIAS. Revista Diálogo Educacional, Curitiba, v. 4, n.12, p.1321, maio/ago. 2004.

VI Congresso Brasileiro de Informática na Educação (CBIE 2017). Anais do XXVIII Simpósio Brasileiro de Informática na Educação (SBIE 2017). Aplicativo para Auxiliar Professores nas Avaliações dePsicogênese da Escrita. MOMBACH, Jaline; SOUZA Paulo Silas; LEAL, Lara.

BRASIL. Secretaria de Educação Básica. Diretoria de Apoio à Gestão Educacional. Pacto nacional pela alfabetização na idade certa : planejamento escolar : alfabetização e ensino da língua portuguesa : ano 1 : unidade 2 / Ministério da Educação, Secretaria de Educação Básica, Diretoria de Apoio à Gestão Educacional. - Brasília : MEC, $2012 . \quad$ Disonível em: $<$ http://www.piraquara.pr.gov.br/aprefeitura/secretariaseorgaos/educacao/upload Address/Unidade_03_Ano_01_\%5B3634\%5D.pdf> . Acesso em : 05 de maio. 2018

ROSSETTO, Alessandra Dedéco Furtado. PSICOGÊNESE DA ESCRITA INFANTIL - PEI. Universidade Tecnológica Federal do Paraná - Campus Londrina Programa de Pós-Graduação Em Ensino de Ciências Humanas, Sociais e da Natureza - Ppgen. 2017

ZANETTE, M. S. Pesquisa qualitativa no contexto da Educação no Brasil. Educar em Revista, Curitiba, Brasil, v. 33, n. 65, p. 149-166, jul./set. 2017. Disponível em:< revistas.ufpr.br/educar/article/download/47454/33236>. Acesso em: 22 maio. 2018. 